\title{
ZYGOTIC GENOTYPIC FREQUENCIES UNDER SELECTION ON FEMALE OR MALE GAMETE PRODUCTION IN PARTIALLY SELF-FERTILIZING PLANT POPULATIONS
}

\author{
MARTIN ZIEHE \\ Lehrstuhl für Forstgenetik und Forstpflanzenzüchtung der Universität Göttingen, \\ Büsgenweg 2, 3400 Göttingen, West Germany
}

Received 6.v.82

\section{SUMMARY}

In order to improve our understanding of the combined influence of mating system components on genotypic structures in zygotes, it may be better to consider the selective effects in each of the two sex functions separately. In this paper, two diallelic selection models for plant populations with mixed selffertilization and random gametic fusion are considered: (A) Selection on the production of free pollen (i.e., pollen not reserved for self-fertilization and thus subject to competition), and (B) selection on the production of ovules (all of which are fertilized). It is well known that sexually asymmetrical fertility selection produces a heterozygotic excess, whereas self-fertilization generally leads to a homozygotic excess relative to Hardy-Weinberg proportions. The combined influence of the two factors is investigated for the diallelic case with special emphasis on three topics: (i) Estimation of limits for the amount of genotypic equilibrium deviations from Hardy-Weinberg proportions, (ii) determination and illustration of the location of genotypic equilibria, and (iii) conditions for protectedness of polymorphisms and alleles. With respect to these aspects, the models $\mathrm{A}$ and $\mathrm{B}$ are compared with the classical symmetrical fertility model.

The results and figures serve to interpret the combined influence of fertility selection and partial self-fertilization on zygotic genotypic structures. In addition, the frequently used argument that in predominantly self-fertilizing organisms only strong overdominance is capable of constituting a polymorphism turns out to require modification.

\section{INTRODUCTION}

THE use of biochemical techniques to determine genotypic structures in zygotic and adult plant populations has become a valuable means of distinguishing between different selective forces (Brown, 1979). Assuming a population reproducing with nonoverlapping generations, the comparison of the genotypic structure of the adults during the reproductive phase with that of their offspring generally neglects the influence of selection for viability. Moreover, this demonstrates the effect of selection acting through reproduction with differential contributions of adult genotypes to the next generation. Nevertheless in the study of natural plant populations it seems to be most unrealistic to try to observe and describe more than just the main reproductive components of the breeding system which lead to a concrete measurable zygotic structure. In this paper we confine our considerations to a combination of two particular reproductive components of a monoecious plant population and to a characterization of their influence on zygotic genotypic structures. These reproductive components are: differential production of pollen or ovules and mixed self-fertilization and random gametic fusion. 
Let us consider the first reproductive component. If the allelic structures are the same in the female and the male gametic pools, then under complete random fusion of gametes the zygotic population will show HardyWeinberg proportions. However, the possibility of having the same allelic distribution in pollen as in ovules is already excluded if genotypes exhibit differential production of female or of male gametes. It has been shown that differential production of ovules and pollen leads immediately to an excess of heterozygotes in the zygotes (Wallace, 1958; Purser, 1966; Ziehe, 1981; Ziehe and Gregorius, 1981). To illustrate this let one homozygote produce predominantly ovules and the other homozygote predominantly pollen; the offspring zygotes will be mainly heterozygous. But as already shown by Ziehe and Gregorius (1981), extreme deviation from HardyWeinberg proportions can be maintained over the generations only if the genotypical gamete production varies extremely asymmetrically in the sexes. With differential production only of pollen or only of ovules, HardyWeinberg equilibria even can result (Ziehe, 1981).

The second reproductive component which will be investigated is partial self-fertilization. It has often been analytically combined with viability or sexually symmetrical fertility selection. Recurrence equations and the corresponding conditions for the computation of equilibria may be found in Hayman (1953), Lewontin (1958), Workman and Jain (1966), Jain and Workman (1967), Woehrmann and Lange (1970) and for the multiallelic case in Weir (1970). Several numerical examples for the development of genotypic structures are presented in Huehn (1979). Partial self-fertilization generally leads to a homozygotic excess which has been well-quantified in the case of no selection. The introduction of selection to the model considerably complicates the immediate estimation of the amount of homozygotic excess in zygotes after several generations or at equilibrium.

The combined influence of partial self-fertilization with differential pollen or ovule production on the zygotic genotypic array has been already investigated in a previous paper (Ziehe, 1982). The main aspects which will be considered here are the heterozygotic deviation from the corresponding Hardy-Weinberg frequency in polymorphic equilibria as a longterm evolutionary effect and criteria for obtaining an idea of the genotypical location of diallelic polymorphic equilibria. Finally, conditions are presented which guarantee the maintenance of an allelic polymorphism.

The effects of differential pollen and ovule production are compared with each other and with the results for models with symmetrical viability or fertility selection and partial self-fertilization.

\section{THE MODELS AND NOTATIONS}

The basic model specified in the present paper is taken from Gregorius and Ross (1981) and refers to an effectively infinite population of diploid monoecious plants reproducing in nonoverlapping generations. A gene locus with alleles $A_{1}$ and $A_{2}$ is considered. Every individual of (unordered) genotype $A_{i} A_{j}$ produces a number $\phi_{i j}$ of ovules from which a proportion $\sigma$ is reserved for self-fertilization and fuses with pollen from the same individual. The remaining part of the pollen, $\mu_{i j}$, is called "free pollen" and enters the free pollen pool of the population. If pollen production is considerably higher than the ovule production, as is generally valid for 
most plant populations, then free pollen production and total pollen production of an individual do not differ appreciably. Every ovule will be fertilized. It is assumed that the probability of fertilizing one of those ovules which are not reserved for self-fertilization is the same for every pollen grain of the free pollen pool (partially random fusion of gametes).

Two models will be discussed in a comprehensive analytical manner and compared with the classical fertility selection model (model C). Model A contains only genotypically differential production of free pollen; model B genotypically differential production of ovules. The parameters for the diallelic case are listed in table 1.

The frequency of those zygotes which contain the alleles $A_{i}$ and $A_{i}$ shall be denoted by $P_{i j}$ (unordered genotypic frequencies) and the allelic frequencies of $A_{1}$ and $A_{2}$ by $p_{1}$ and $p_{2}$, respectively. It will prove to be useful to introduce the average adult allelic contributions $\bar{\mu}_{i}$ and $\bar{\phi}_{i}$ of the allele $A_{i}$ to free pollen and ovule production, respectively. The definitions are valid only if $0<p_{1}, p_{2}<1$ :

$$
\bar{\mu}_{1}=\frac{P_{11}}{p_{1}} \mu_{11}+\frac{P_{12} / 2}{p_{1}} \mu_{12}, \quad \bar{\mu}_{2}=\frac{P_{22}}{p_{2}} \mu_{22}+\frac{P_{12} / 2}{p_{2}} \mu_{12}
$$

and

$$
\bar{\phi}_{1}=\frac{P_{11}}{p_{1}} \phi_{11}+\frac{P_{12} / 2}{p_{1}} \phi_{12}, \quad \bar{\phi}_{2}=\frac{P_{22}}{p_{2}} \phi_{22}+\frac{P_{12} / 2}{p_{2}} \phi_{12} .
$$

The average productions $\bar{\mu}$ and $\bar{\phi}$ of pollen and ovules for $0<p_{1}, p_{2}<1$ are

$$
\bar{\mu}=p_{1} \bar{\mu}_{1}+p_{2} \bar{\mu}_{2} \text { and } \bar{\phi}=p_{1} \bar{\phi}_{1}+p_{2} \bar{\phi}_{2} \text {. }
$$

Parameters denoting the succeeding generation are indicated by primes.

\section{Model A: Selection on pollen production}

\section{(i) The recurrence equations}

The recurrence equations connecting the zygotic genotypic structures over the generations under selection in free pollen production read (compare e.g., Gregorius and Ross, 1981)

$$
\begin{aligned}
& P_{11}^{\prime}=\sigma\left(P_{11}+\frac{P_{12}}{4}\right)+(1-\sigma) p_{1}^{2} \frac{\bar{\mu}_{1}}{\bar{\mu}} \\
& P_{12}^{\prime}=\sigma \frac{P_{12}}{2}+2(1-\sigma) p_{1} p_{2}\left(\frac{\bar{\mu}_{1}+\bar{\mu}_{2}}{2 \bar{\mu}}\right)
\end{aligned}
$$

and

$$
P_{22}^{\prime}=\sigma\left(P_{22}+\frac{P_{12}}{4}\right)+(1-\sigma) p_{2}^{2} \frac{\bar{\mu}_{2}}{\bar{\mu}} \text { for } 0 \neq P_{1} \neq 1
$$

Considering the heterozygous zygotic frequency of the subsequent generation, we know (Ziehe, 1982)

$$
P_{12}^{\prime} \geqq\left[\sigma \frac{P_{12}}{2}+2\left(\frac{1-\sigma}{1+\sigma}\right) p_{1}^{\prime} p_{2}^{\prime}\right] .
$$



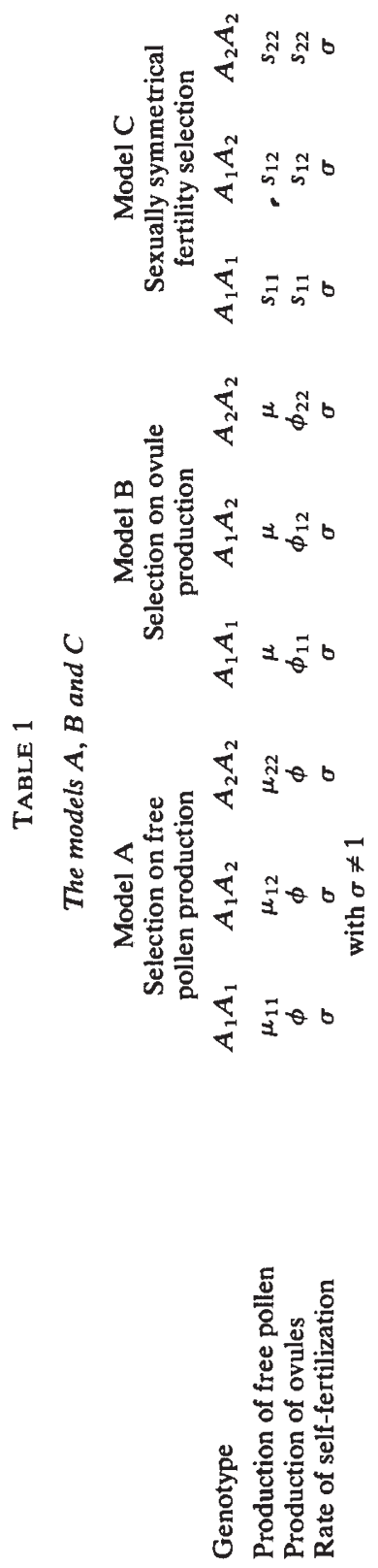
Thus, even if heterozygotes which again produce heterozygotes by selffertilization are not present in the preceding generation, the total heterozygotic frequency after the first generation will lie on or above a reduced Hardy-Weinberg parabola calculated from $2 p_{1} p_{2}((1-\sigma) /(1+\sigma))$. Definining the panmictic index $I_{p}$ by $I_{p}=P_{12} / 2 p_{1} p_{2}$, this corresponds to $I_{p}$ greater than $(1-\sigma) /(1+\sigma)$ after the first generation. Using Wright's fixation index $F, F=1-I_{p}$, as a measure for the departure from Hardy-Weinberg proportions, the resulting genotypic structures show $F \leqq 2 \sigma /(1-\sigma)$ after one generation at the latest. In the special case of complete random fusion of the gametes produced $(\sigma=0)$, this is equivalent to the wellknown heterozygotic excess relative to Hardy-Weinberg proportions for selection acting only in one sex (Purser, 1966; Ziehe, 1981; Ziehe and Gregorius, 1981).

On the other hand, Ziehe (1982) presents an upper limit for the panmictic index namely $I_{p} \leqq 2 /(1+\sigma)$. This is equivalent to a lower limit for the fixation index: $F \geqq-(1-\sigma) /(1+\sigma)$. Examples of selection regimes which strongly favour the pollen production of one homozygote in fact demonstrate that for $\sigma=0$ an arbitrarily high level of offspring heterozygosity may be reached provided a suitable adult genotypic structure is chosen.

\section{(ii) The change in allelic frequencies}

From the above genotypic recurrence equations, the allelic recurrerce equation for $p_{1}$ reduces to

$$
p_{1}^{\prime}=\sigma p_{1}+(1-\sigma) p_{1}\left(\frac{1}{2}+\frac{1}{2} \frac{\bar{\mu}_{1}}{\bar{\mu}}\right)=p_{1}\left[\frac{1+\sigma}{2}+\frac{1-\sigma}{2} \frac{\bar{\mu}_{1}}{\bar{\mu}}\right] .
$$

Thus the direction of change for all allelic frequencies is independent of $\sigma$ and only depends on whether $\bar{\mu}_{1}$ is less than, equal to or greater than $\bar{\mu}$. In particular, the occurrence of allelic constancy between two successive generations $\left(p_{1}^{\prime}=p_{1}\right.$ and $\left.p_{2}^{\prime}=p_{2}\right)$ is equivalent to $\bar{\mu}_{1}=\bar{\mu}_{2}=\bar{\mu}$. A discussion of the set of genotypic frequencies with $\bar{\mu}_{1}=\bar{\mu}_{2}=\bar{\mu}$ is given in Ziehe (1981).

Special cases with globally describable dynamics are:

(a) The case of no selection $\left(\mu_{11}=\mu_{12}=\mu_{22}\right)$. For every genotypic structure except the fixation points we have $\bar{\mu}_{1}=\bar{\mu}_{2}=\bar{\mu}$ and therefore no allelic change (compare Hayman, 1953).

(b) The intermediate cases $\mu_{11}<\mu_{12}<\mu_{22}$ and $\mu_{22}<\mu_{12}<\mu_{11}$. The sole point for which $\bar{\mu}_{1}=\bar{\mu}_{2}=\bar{\mu}$ is the genotypic structure consisting only of heterozygous individuals. Thus $p_{1}$ changes, if at all, consistently in one direction until fixation of that allele occurs for which the homozygotic genotype has the highest pollen production.

(c) The case of allelic selective symmetry $\mu_{11}=\mu_{22} \neq \mu_{12}$. Here $\bar{\mu}_{1}=$ $\bar{\mu}_{2}=\bar{\mu}$ is valid if either $p_{1}=p_{2}=0.5$ or there exists no heterozygous individual $\left(P_{12}=0\right)$. With overdominance, all genotypic structures (except fixation points) converge to a polymorphic equilibrium in $\hat{p}_{1}=0.5$ and $\hat{P}_{12}=(1-\sigma) /(2-\sigma)$. In the case of underdominance, convergence is to fixation of that allele with the higher initial allelic frequency (see Appendix 1 ), except from $p_{1}=0 \cdot 5$. For initial $p_{1}=0.5, p_{1}$ remains constant and $P_{12}$ again converges to $\hat{P}_{12}=(1-\sigma) /(2-\sigma)$.

In all other cases, a graphical description of the set of genotypic frequencies for which $\bar{\mu}_{1}=\bar{\mu}_{2}=\bar{\mu}$ (not defined for fixation points) can be presented 
by plotting the heterozygotic frequency versus $p_{1}$ in a DeFinetti diagram. The associated function is

$$
P_{12}=2 p_{1} p_{2} \frac{1-\gamma}{1-p_{1}(1+\gamma)}
$$

where

$$
\gamma=\frac{\mu_{12}-\mu_{22}}{\mu_{12}-\mu_{11}}
$$

measures the selective asymmetry between the homozygous genotypes relative to the heterozygous. It is a monotone function (increasing for $\mu_{12} \leqq \mu_{22}<\mu_{11}$ and $\mu_{11}<\mu_{22} \leqq \mu_{12}$ and decreasing for $\mu_{12} \leqq \mu_{11}<\mu_{22}$ and $\mu_{22}<\mu_{11} \leqq \mu_{12}$ ), connecting one of the fixation points with the structure of a totally heterozygous population. Examples for several fixed values of $\gamma$ are illustrated in fig. 1.

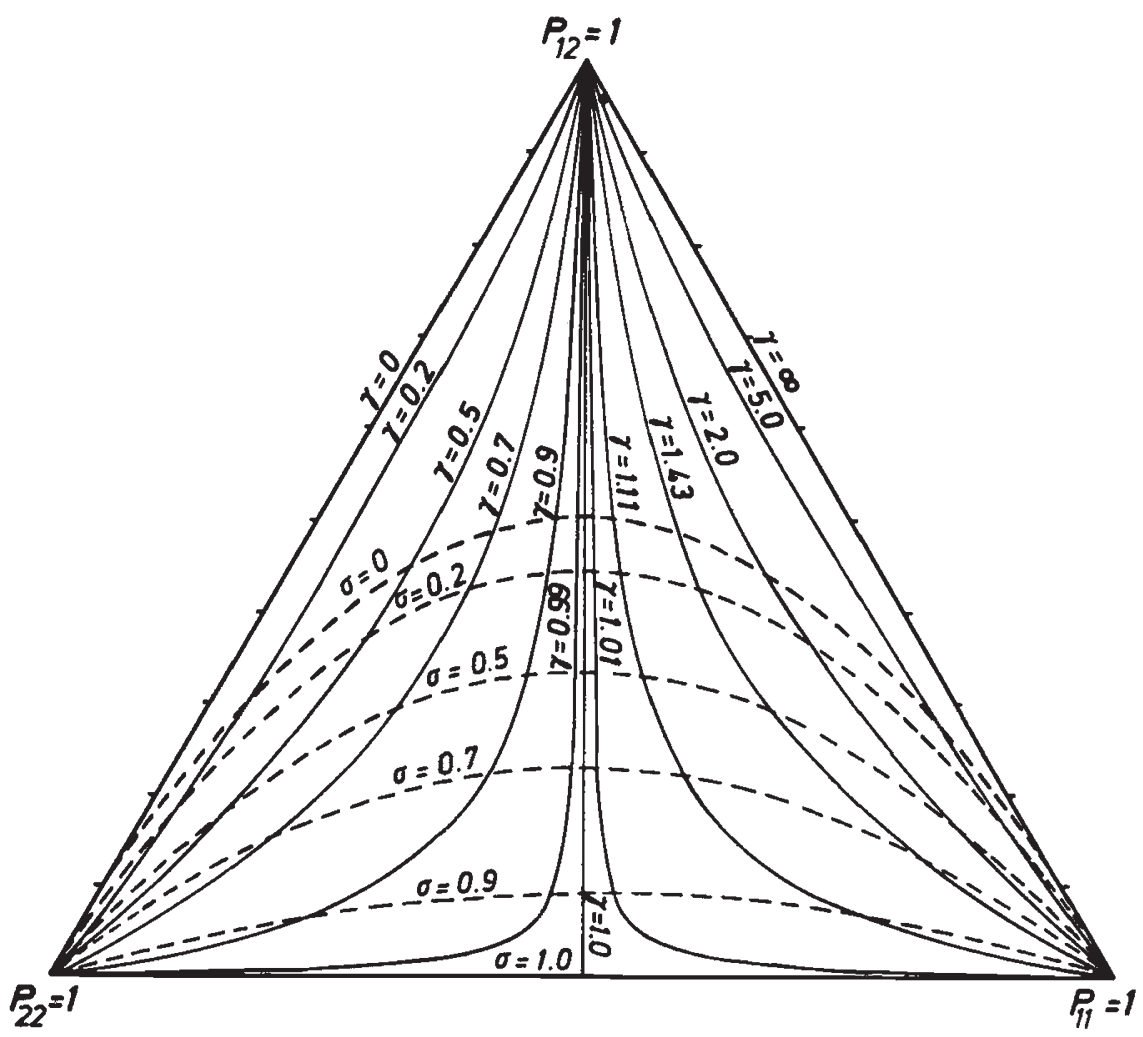

FIG. 1.-The location of equilibria for model A, depending on the selective asymmetry parameter $\gamma$ and the self-fertilization rate $\sigma$.

(iii) The location of nontrivial equilibria

If in addition to the allelic constancy the heterozygotic frequency does not change, an equilibrium is reached. Provided that $\bar{\mu}_{1}=\bar{\mu}_{2}=\bar{\mu}$, the 
equality $P_{12}^{\prime}=P_{12}$ is valid only if (resulting from the recurrence equation)

$$
P_{12}=2 p_{1} p_{2} \frac{1-\sigma}{1-(\sigma / 2)} .
$$

Thus the deficiency of heterozygotes at equilibrium is independent of the selection regime on free pollen and refers to the panmictic index $I_{p}=$ $(1-\sigma) /[1-(\sigma / 2)]$ or the fixation index $F=\sigma /(2-\sigma)$. This is therefore the same panmictic index as for a system without selection (compare Hayman 1953): Selection in pollen production does not affect the HardyWeinberg deficiency at equilibrium. In the special case of $\sigma=0$, this implies Hardy-Weinberg equilibria even in the presence of selection in pollen production, a fact which is already known. The graphs of genotypic frequencies with $(* *)$ are illustrated as dotted lines (depending on $\sigma$ ) in fig. 1.

A nontrivial equilibrium occurs at the intersection of the lines described by $(*)$ and $(* *)$, which reflect the selection ( $\gamma$-curve) and the self-fertilization influence ( $\sigma$-curve), respectively. Fig. 1 therefore shows the location of genotypic equilibria under the combined influences. For a fixed selection regime represented by the parameter $\gamma$ and for increasing $\sigma$, the equilibrium point moves downwards along the $\gamma$-line until it reaches the fixation point.

If a nontrivial equilibrium exists, it exhibits the allelic frequency

$$
\hat{p}_{1}=\frac{(1-\sigma)-(1-\gamma)(1-\sigma / 2)}{(1+\gamma)(1-\sigma)} .
$$

As is suggested in fig. 1, a polymorphic intersection need not exist. Assuming $\gamma<1$, there is no nontrivial equilibrium if $\gamma<\sigma /(2-\sigma)$; for $\gamma>1$ none exists if $\gamma>(2-\sigma) / \sigma$.

The case $\gamma=1$ of allelic selective symmetry $\left(\mu_{11}=\mu_{22} \neq \mu_{12}\right.$, case (c) of the preceding section) always produces a nontrivial equilibrium.

\section{(iv) Conditions for protectedness of polymorphisms}

The last section for the model of differential pollen production and partial self-fertilization refers to conditions under which no allele can be lost within the population or even stay at an arbitrarily low frequency for an indefinite number of generations, provided all genotypes are present initially. This in particular implies instability of the fixation points. Conditions for allelic protectedness for a more general model are presented in Gregorius (1982a) (compare Appendix 2) and will be applied to the present model. We assume that selection acts on pollen production such that $\mu_{11}$, $\mu_{12}$ and $\mu_{22}$ are not equal; otherwise there is no allelic change.

Applying both inequalities of Appendix 2 to this model, they reduce to a single condition for the protection of $A_{1}$ :

$$
(1-\sigma) \mu_{12}+\frac{\sigma}{2} \mu_{11}>\left(1-\frac{\sigma}{2}\right) \mu_{22} .
$$

To obtain the condition for protectedness of $A_{2}$, the indices 1 and 2 must be interchanged. 
Making use of the classification into overdominant, underdominant and intermediate or dominant pollen production, $\boldsymbol{A}_{1}$ for example is protected if

(a) $\mu_{12}>\mu_{11}, \mu_{22}$ and $\gamma>\frac{\sigma}{2-\sigma}\left(\right.$ or $\left.\sigma<\frac{2 \gamma}{1+\gamma}\right)$, or if

(b) $\mu_{12}<\mu_{11}, \mu_{22}$ and $\gamma<\frac{\sigma}{2-\sigma}\left(\right.$ or $\left.\sigma>\frac{2 \gamma}{1+\gamma}\right)$, or if

(c) $\mu_{22} \leqq \mu_{12} \leqq \mu_{11}$ and not all equal.

It should be mentioned that the conditions for each of the three cases depend only on the asymmetry coefficient $\gamma$ and the self-fertilization rate $\sigma$.

Both alleles are protected simultaneously and thus both fixation points are unstable in the case of overdominance when in addition

$$
\frac{\sigma}{2-\sigma}<\gamma<\frac{2-\sigma}{\sigma}
$$

which places a restriction on the selective asymmetry coefficient.

Thus for overdominance, the existence of a nontrivial equilibrium is combined with a protected polymorphism and unstable fixation points. This in fact is no proof for stability of or even global convergence to the polymorphic equilibrium, both resulting in hardly surveyable algebraic expressions. But up to now, all computer simulations of genotypic trajectories confirm the hypothesis of convergence. For the special case $\sigma=0$ but arbitrary pollen production, the gloval convergence to (Hardy-Weinberg) equilibria has already been shown (Ziehe, 1981).

\section{Model B: Selection on ovule production}

\section{(i) The recurrence equations}

For the alternative model of selection on ovule production, the recurrence equations have already been determined by Workman and Jain (1966). Using our notation, we have

and

$$
\begin{aligned}
& P_{11}^{\prime}=\sigma\left(\frac{\phi_{11} P_{11}+\phi_{12} P_{12} / 4}{\bar{\phi}}\right)+(1-\sigma) p_{1}^{2} \frac{\bar{\phi}_{1}}{\bar{\phi}} \\
& P_{12}^{\prime}=\sigma \frac{\phi_{12} P_{12} / 2}{\bar{\phi}}+2(1-\sigma) p_{1} p_{2}\left(\frac{\bar{\phi}_{1}+\bar{\phi}_{2}}{2 \bar{\phi}}\right),
\end{aligned}
$$

$$
P_{22}^{\prime}=\sigma\left(\frac{\phi_{22} P_{22}+\phi_{12} P_{12} / 4}{\bar{\phi}}\right)+(1-\sigma) p_{2}^{2} \frac{\bar{\phi}_{2}}{\bar{\phi}} \text { for } 0 \neq p_{1} \neq 1 \text {. }
$$

A previous paper (Ziehe, 1982) contains the derivaticn of a lower limit for the heterozygotic frequency after one generation at the latest,

$$
P_{12}^{\prime} \geqq \sigma \frac{P_{12}}{2} \frac{\phi_{12}}{\bar{\phi}}+2\left(\frac{1-\sigma}{1+\sigma}\right) p_{1}^{\prime} p_{2}^{\prime},
$$

which leads again to a panmictic index greater than $(1-\sigma) /(1+\sigma)$ or a fixation index less than $2 \sigma /(1-\sigma)$. 
Again $2 /(1+\sigma)$ is an upper limit for the panmictic index, $-(1-\sigma) /(1+$ $\sigma)$ a lower limit for the fixation index (Ziehe, 1982).

(ii) The change in allelic frequencies

The allelic recurrence equation reads

$$
p_{1}^{\prime}=\sigma p_{1} \frac{\bar{\phi}_{1}}{\bar{\phi}}+(1-\sigma) p_{1}\left(\frac{1}{2}+\frac{\bar{\phi}_{1}}{2 \bar{\phi}}\right)=p_{1}\left[\frac{1+\sigma}{2} \frac{\bar{\phi}_{1}}{\bar{\phi}}+\frac{1-\sigma}{2}\right]
$$

for $0 \neq p_{1}$. Therefore here again $p_{1}^{\prime}=p_{1}$ if and only if $\bar{\phi}_{1}=\bar{\phi}_{2}=\bar{\phi}$ independently of the rate of self-fertilization. Thus denoting the selective asymmetry coefficient as before by $\gamma, \gamma=\left(\phi_{12}-\phi_{22}\right) /\left(\phi_{12}-\phi_{11}\right)$, we obtain the same analytic structure as in model $\mathrm{A}$ for the set of genotypic frequencies with allelic constancy between two successive generations (namely the $\gamma$-curves of fig. 1).

\section{(iii) The location of equilibria}

The determination of the equilibria by equating heterozygotic frequencies over the generations under $\bar{\phi}_{1}=\bar{\phi}_{2}=\bar{\phi}$ leads to

$$
P_{12}=2 p_{1} p_{2}\left[\frac{1-\sigma}{1-(\sigma / 2) \phi_{12} / \bar{\phi}}\right] \text {. }
$$

The frequency-dependence of the equilibrium panmictic index may be eliminated, which leads to the complicated formula for the equilibrium allelic frequencies presented in Workman and Jain (1966). These do not provide any clues as to the location of the equilibrium. Nevertheless, it is possible to give structurally simple limits for the panmictic index which are frequency-independent.

\section{(a) Heterozygotic advantage: $\phi_{12} \geqq \phi_{11}, \phi_{22}$}

Under heterozygotic advantage, we have $\phi_{12} \geqq \bar{\phi}$ for every genotypic structure and therefore the panmictic index $I_{p}=(1-\sigma) /\left[1-(\sigma / 2) \phi_{12} / \bar{\phi}\right]$ is always greater than or equal to $(1-\sigma) /(1-\sigma / 2)$.

Assuming a genotype structure with $\bar{\phi}_{1}=\bar{\phi}_{2}=\bar{\phi}$ lying above HardyWeinberg proportions $\left(P_{12}>2 p_{1} p_{2}\right)$, we have

$$
\bar{\phi}=\bar{\phi}_{2} \geqq \frac{P_{12} / 2}{1-p_{1}} \phi_{12} \geqq p_{1} \phi_{12} \geqq \frac{1}{2} \phi_{12} \quad \text { for } p_{1} \geqq \frac{1}{2}
$$

and

$$
\bar{\phi}=\bar{\phi}_{1} \geqq \frac{P_{12} / 2}{p_{1}} \phi_{12} \geqq\left(1-p_{1}\right) \phi_{12} \geqq \frac{1}{2} \phi_{12} \quad \text { for } p_{1} \leqq \frac{1}{2} .
$$

Thus $2 \bar{\phi} \geqq \phi_{12}$. But this is compatible only with an equilibrium panmictic index $I_{p}$ less than or equal to 1 , which contradicts the assumption of an equilibrium located above Hardy-Weinberg frequencies. Thus an equilibrium under selection only on ovules cannot exceed the upper limit of Hardy-Weinberg proportions. 
(b) Heterozygotic disadvantage: $\phi_{12} \leqq \phi_{11}, \phi_{12}$

Since $\phi_{12} \leqq \bar{\phi}$, the panmictic index $I_{p}=(1-\sigma) /\left(1-(\sigma / 2)\left(\phi_{12} / \bar{\phi}\right)\right)$ possess the upper limit $(1-\sigma) /(1-\sigma / 2)$. On the other side, $(1-\sigma)$ is evidently a lower limit for the equilibrium panmictic index.

The equilibrium location is as follows. Provided there is selection, nontrivial equilibria can only exist in the cases described of heterozygotic advantage or disadvantage.

For heterozygotic advantage $\left(\phi_{12} \geqq \phi_{11}, \phi_{22}\right)$ an equilibrium lies on the respective $\gamma$-curve of fig. 1 between its intersection with the Hardy-Weinberg parabola and its intersection with the curve of the panmictic index $(1-\sigma) /(1-\sigma / 2)$ (see fig. 1). For a large heterozygote advantage, HardyWeinberg proportions approximately may be reached. For heterozygotic disadvantage $\left(\phi_{12} \leqq \phi_{11}, \phi_{22}\right)$ an equilibrium lies on the respective $\gamma$-curve between its intersection with the curve of the panmictic index $(1-\sigma) /(1-$ $\sigma / 2)$ and that of $(1-\sigma)$. Clearly, if both intersections occur in the fixation point, there is no possibility for a polymorphic equilibrium to exist.

\section{(iv) Conditions for protectedness of polymorphisms}

An application of the inequalities of Appendix 2 to a model with only differential ovule production (and thus $\mu_{i j}=\mu$ ) and $\sigma \neq 1$ leads to protection of $A_{1}$ (and thus in particular to instability of the fixation point with $p_{1}=0$ ) if

$$
\frac{\sigma}{2} \frac{\phi_{12}}{\phi_{22}}\left(\frac{\phi_{11}}{\phi_{22}}-1\right)>\left(1-\sigma \frac{\phi_{11}}{\phi_{22}}\right)\left(1-\frac{\phi_{12}}{\phi_{22}}\right) .
$$

This is fulfilled if

(a) $\phi_{12}>\phi_{11}, \phi_{22}$ and either

(i) $\phi_{11} \geqq \phi_{22}$ or

(ii) $\phi_{22}<\phi_{11}$ and

or if

$$
\gamma>\frac{\sigma}{2-\sigma\left(\phi_{11} / \phi_{22}\right)}\left(\text { i.e. } \sigma<\frac{2 \gamma}{1+\gamma\left(\phi_{11} / \phi_{22}\right)}\right),
$$

(b) $\phi_{12}<\phi_{22} \leqq \phi_{11}$ and

or if

$$
\gamma<\frac{\sigma}{2-\sigma\left(\phi_{11} / \phi_{22}\right)}\left(\text { i.e. } \sigma>\frac{2 \gamma}{1+\gamma\left(\phi_{11} / \phi_{22}\right)}\right),
$$

(c) $\phi_{22} \leqq \phi_{12} \leqq \phi_{11}$ and not all equal.

The diallelic polymorphism (and thus each of the alleles) is protected in the case of overdominance when in addition

or

$$
\phi_{11} \leqq \phi_{22} \quad \text { and } \quad \gamma>\frac{\sigma}{2-\sigma\left(\phi_{11} / \phi_{22}\right)}
$$

$$
\phi_{22} \leqq \phi_{11} \quad \text { and } \quad \gamma<\frac{2-\sigma\left(\phi_{22} / \phi_{11}\right)}{\sigma}
$$


Evidently, the conditions of model $\boldsymbol{A}$ for selection in pollen production, namely overdominance and $\sigma /(2-\sigma)<\gamma<(2-\sigma) / \sigma$, are sufficient for polymorphism protectedness in this model. For an illustration using phase diagrams compare fig. 4.

\section{A NOTE ON SYMMETRICAL FERTILITY SELECTION AND VIABILITY SELECTION (MODEL C)}

Symmetrical fertility selection with coefficients $s_{i j}=\phi_{i j}=\mu_{i j}$ (or viability selection with a survival probability $s_{i j}$ ) of the genotype $A_{i} A_{j}$ yields the following recurrence equation for the heterozygous zygote frequency (provided $0 \neq p_{1} \neq 1$ ):

$$
\begin{aligned}
P_{12}^{\prime} & =\sigma \frac{s_{12}}{\bar{s}} \frac{P_{12}}{2}+2(1-\sigma) p_{1} p_{2} \frac{\bar{s}_{1} \bar{s}_{2}}{\bar{s}_{2}^{2}} \\
& =\sigma \frac{s_{12}}{\bar{s}} \frac{P_{12}}{2}+2(1-\sigma) p_{1}^{\prime} p_{2}^{\prime},
\end{aligned}
$$

where

$$
\bar{s}_{i}=\frac{P_{i i}}{p_{i}} s_{i i}+\frac{P_{12} / 2}{p_{i}} s_{12}, \quad p_{i}^{\prime}=P_{i} \bar{s}_{i} / \bar{s} \quad(i=1,2)
$$

and

$$
\bar{s}=p_{1} \bar{s}_{1}+p_{2} \bar{s}_{2}
$$

Thus after one generation at the latest, the panmictic index $I_{p}$ is greater than $(1-\sigma)$. Additionally, as proven in Ziehe (1982), a homozygotic excess relative to Hardy-Weinberg proportions occurs in the offspring generation, which implies $I_{p}<1$ after one generation at the latest.

In equilibrium we have the same structure as for selection in ovule production, as already shown by Workman and Jain (1966) $\left(\bar{s}_{1}=\bar{s}_{2}=\bar{s}\right.$ and panmictic index $\left.(1-\sigma) /\left(1-(\sigma / 2)\left(s_{12} / \bar{s}\right)\right)\right)$.

Therefore the discussion of the location of equilibria is analogous to that for model B.

The analysis of protectedness of alleles and the diallelic polymorphism leads analytically to the same conditions as for model B, replacing $\phi_{i j}$ by $s_{i j}$. The corresponding condition for a protected polymorphism coincides with that derived by Kimura and Ohta (1971) regarding instability of both fixation points simultaneously.

\section{A COMPARISON OF THE MODELS AND A SUMMARY OF THE RESULTS}

In the preceding sections, several selection models for partially selffertilizing plant populations were considered. Limits for the deviations from Hardy-Weinberg proportions, depending in the last analysis only on the rate $\sigma$ of self-fertilization, were presented in terms of panmictic and fixation indices.

Details and additional results for symmetrical fertility selection are recapitulated in table 2 . 

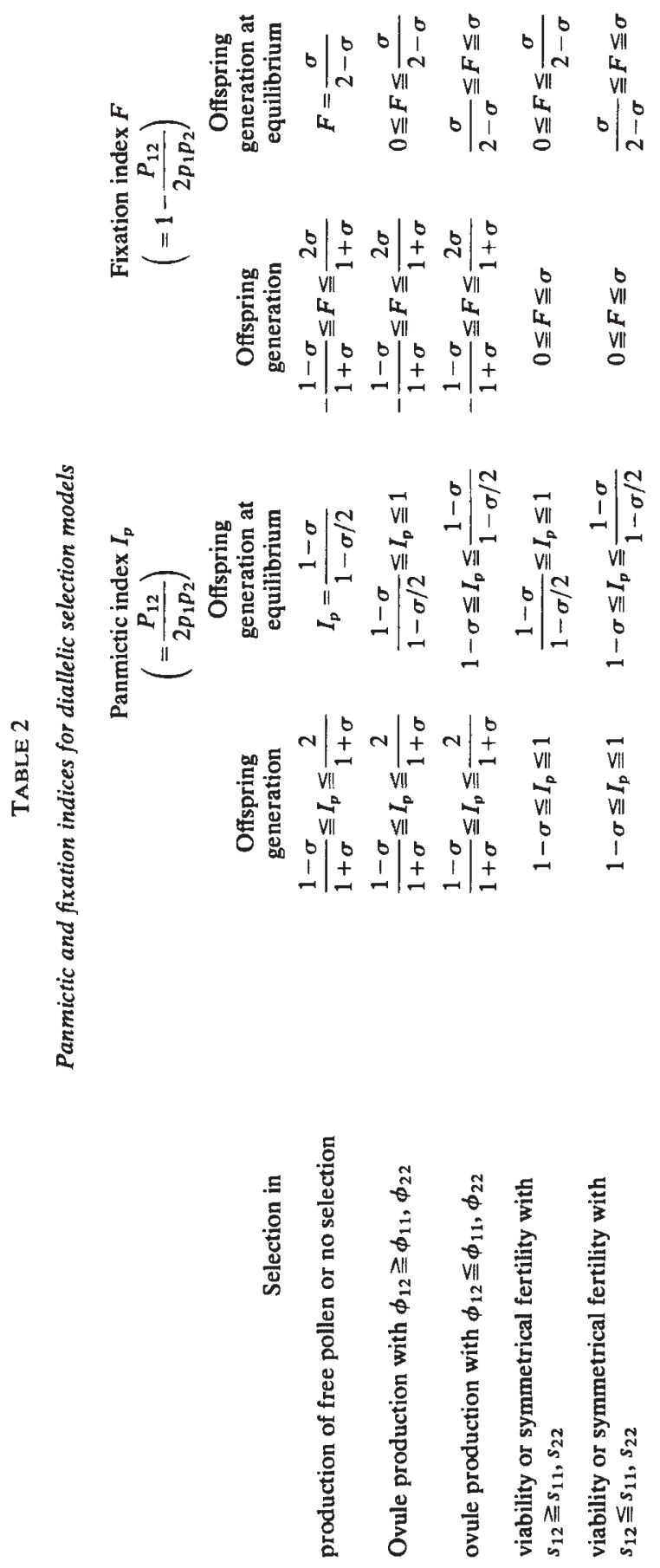


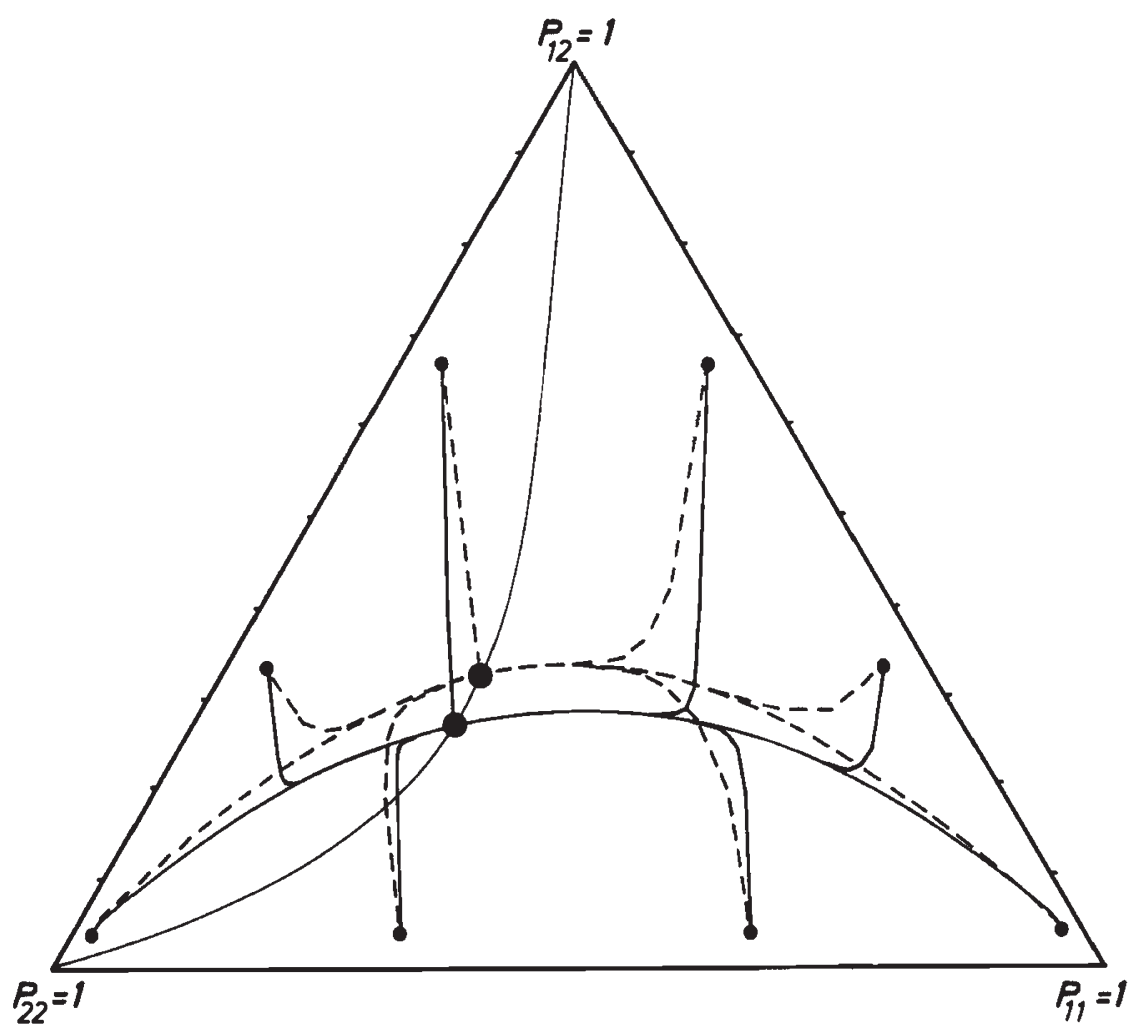

FIG. 2.-Genotypic trajectories for eight different starting points under two selection regimes and $\sigma=0.6$. The dotted lines refer to selection on ovule production with $\phi_{11}=0.8$, $\phi_{12}=1.4, \phi_{22}=0.9$, the solid lines to selection on production of free pollen with $\mu_{11}=0.8$, $\mu_{12}=1.4, \mu_{22}=0.9$. The figure also contains the $\gamma$-curve with the equilibria.

The limits for the panmictic and fixation indices in the offspring generation are valid for all selection regimes which refer to the respective selection model. Numerical simulations show that if the intensity of selection is weak (small differences in gamete production relative to their genotypic mean), then the ultimate panmictic index approximately will be reached after only a few generations, whereas the allelic frequencies may change slowly. Fig. 2 contains an example which illustrates this.

Referring to the discussion about the change in allelic frequencies, we arrive at the following results:

Result 1: The analytical investigations of the selection models presented prove that the direction of allelic change from the parental generation to the offspring is independent of the rate of self-fertilization, $\sigma$. The regions of increasing or decreasing allelic frequency are separated by the $\gamma$-curves of fig. 1. Moreover, these $\gamma$-curves are constructed in an analytically equivalent manner for all selection models under consideration, using only the selection coefficients.

A broader discussion of the importance of these $\gamma$-curves is presented in Gregorius (1982b). Since equilibria must lie on the respective $\gamma$-curve, the limitations of the equilibrium panmictic indices of table 2 yield a useful 
localization of polymorphic equilibria:

Result 2: An equilibrium point for model A with differential free pollen production occurs at the intersection of the respective $\gamma$-curve and the $\sigma$-parabola in fig. 1. Equilibria for model B of differential ovule production and for models of symmetrical fertility selection are located on the segment of the $\gamma$-curve between the intersections with the Hardy-Weinberg parabola and the $\sigma$-parabola in the case of overdominance in the selection coefficients. They are located on the $\gamma$-curve below the intersection with the $\sigma$-parabola for underdominance.

Therefore for $0<\sigma<1$, a selection regime with heterozygotic advantage for the last-mentioned models leads to a higher heterozygote frequency in equilibrium than the same selection regime for differential production of free pollen. For extreme heterozygotic advantage under symmetrical fertility selection or differential ovule production, even Hardy-Weinberg proportions may be reached. The amount of equilibrium heterozygotic advantage or disadvantage for models with allelic selective symmetry $(\gamma=1)$ is illustrated in fig. 3. It should be emphasized that convergence to this equilibrium occurs for overdominance. The corresponding equilibrium formula is taken from Appendix 1. $d$ measures the relative additional advantage or disadvantage of the heterozygotic selection coefficient compared with the homozygote; for example for model C: $s_{12}=(1+d) s_{\mathrm{Hom}}$.

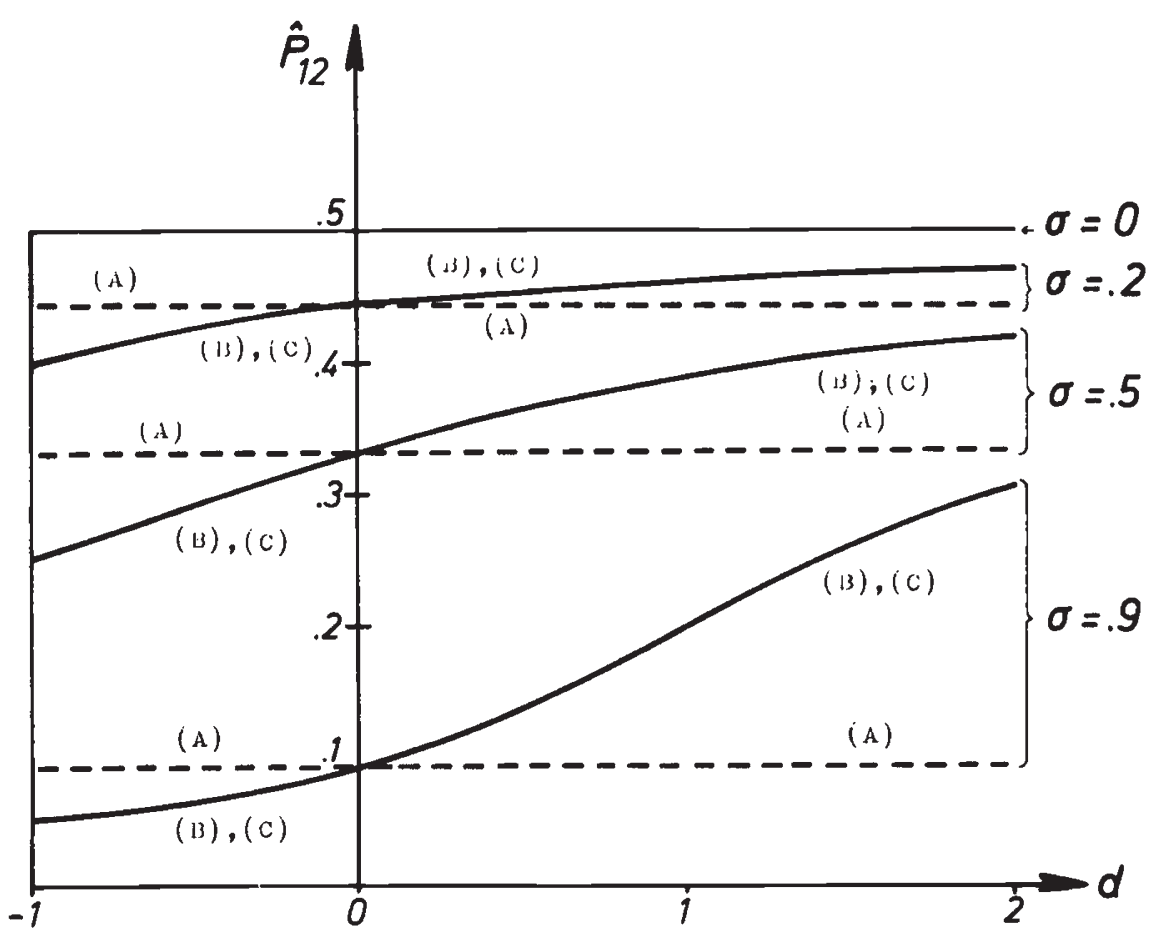

FIG. 3.-Heterozygote equilibrium frequency for models with allelic selective symmetry and thus allelic equilibrium frequency $\hat{p}_{1}=0 \cdot 5$. (A) refers to differential pollen production with $\mu_{11}=\mu_{22}=\mu_{\text {Hom }}$ and $d=\left(\mu_{12}-\mu_{\text {Hom }}\right) / \mu_{\text {Hom }}$, (B) to differential ovule production with $\phi_{11}=\phi_{22}=\phi_{\text {Hom }}$ and $d=\left(\phi_{12}-\phi_{\text {Hom }}\right) / \phi_{\text {Hom }}$ and (C) to differential sexually symmetrical viability or fertility selection with $s_{11}=s_{22}=s_{\mathrm{Hom}}$ and $d=\left(s_{12}-s_{\mathrm{Hom}}\right) / s_{\mathrm{Hom}}$. 


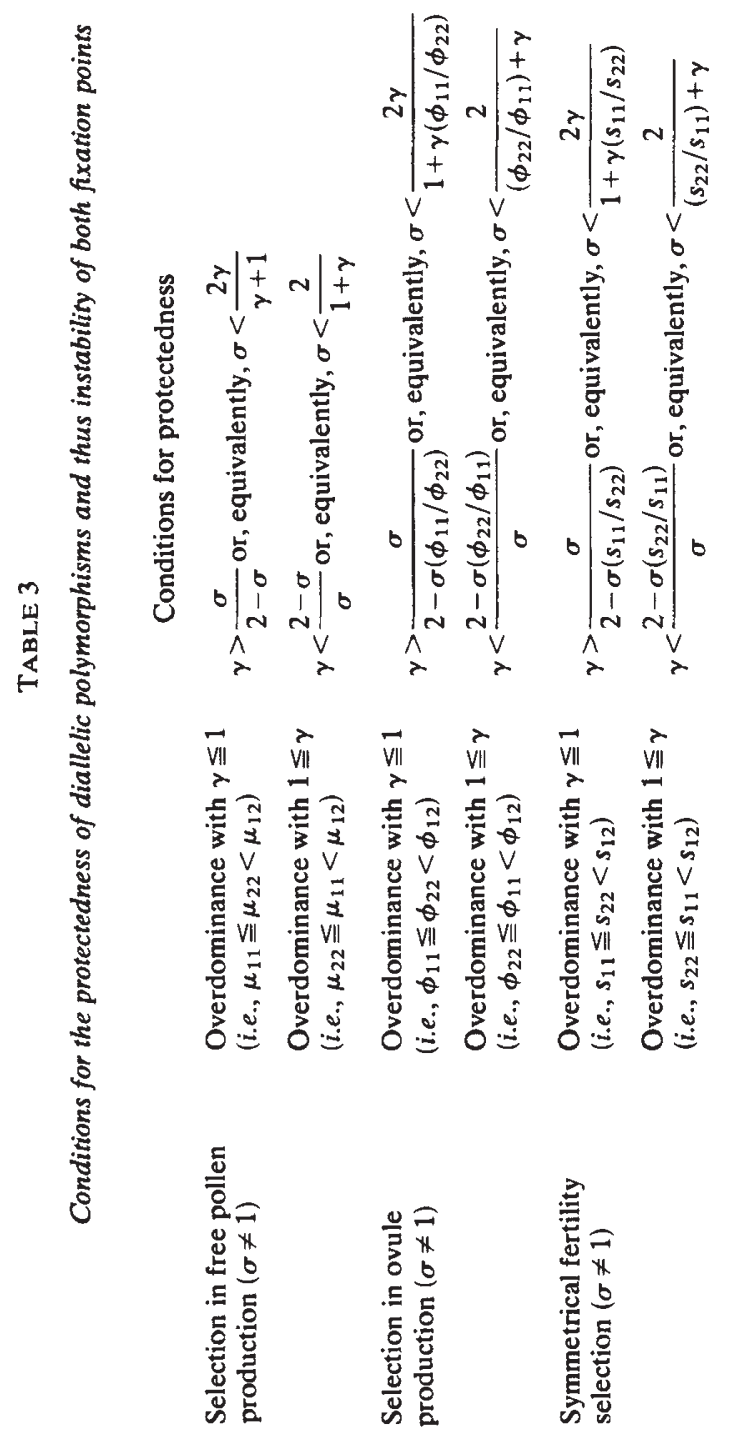



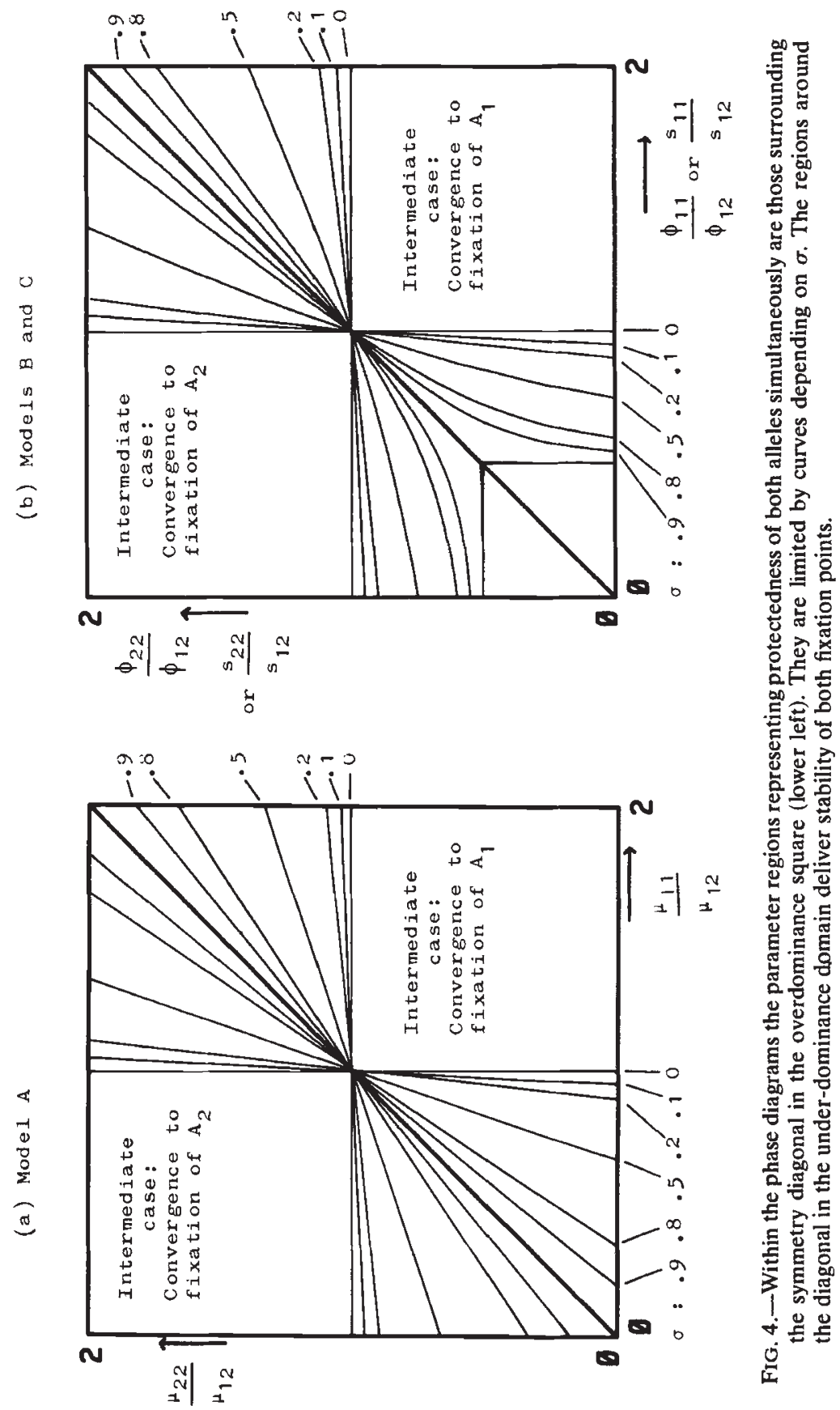
Overdominance has proved to be a necessary condition for simultaneous protectedness of both alleles, and thus of the polymorphism, in all selection models under consideration.

Result 3: In the case of differential pollen production, the protectedness of both alleles (and thus instability of their fixation points and existence of a polymorphic equilibrium) requires overdominance and an increasing symmetry in homozygotic disadvantage $(\gamma \rightarrow 1)$ for an increasing rate of self-fertilization $(\sigma \rightarrow 1)$. In the case of differential ovule production or symmetrical fertility selection the conditions for the protectedness of the polymorphism permit more asymmetry in homozygotic disadvantage for weak overdominance. Strong overdominance as with $\phi_{11}, \phi_{22}<$ $(1-\sigma / 2) \phi_{12}$ or $s_{11}, s_{22}<(1-\sigma / 2) s_{12}$, respectively, guarantees a protected polymorphism without restrictions.

The exact conditions for protectedness of polymorphism are summarized in table 3 and illustrated in fig. 4.

Several authors in recent articles have adopted the claim of Kimura and Ohta (1971), who stated for the symmetrical fertility selection model that "overdominance cannot be a major mechanism for polymorphism in predominantly self-fertilizing organisms, unless the overdominance is very strong". It has been shown in this paper that, in the case of overdominance, those conditions for the protectedness of the polymorphism and the existence of a polymorphic equilibrium for model $A$ of differential free pollen production are sufficient conditions for each of the two other selection models, if we assume the same selection coefficients. Figs. 1 and 4 immediately show that for a high rate of self-fertilization and overdominance a polymorphic equilibrium for model $\mathrm{A}$ exists and the polymorphism is protected if $\gamma$ is near to 1 . In the extreme case $\gamma=1\left(\mu_{11}=\mu_{22}<\mu_{12}\right)$, a polymorphic equilibrium exists and the polymorphism is protected for every $\sigma<1$. Moreover in this special case, as proved in Appendix 1, all genotypic frequencies except from fixation converge to the polymorphic equilibrium. Thus for predominantly self-fertilizing organisms the main mechanism for establishing polymorphisms for selection models with overdominance is not only the amount of heterozygotic advantage, but also the symmetry in homozygotic disadvantage expressed as small deviations of $\gamma$ from 1.

Acknowledgements.-These investigations were partially supported by a grant from the Deutsche Forschungsgemeinschaft. The author is indebted to H.-R. Gregorius for helpful advice, to E, Gillet for the drawings and to B. Peter for her patience in typing the manuscript.

\section{REFERENCES}

BROWN, A. H. D. 1979. Enzyme polymorphism in plant populations. Theoretical Population Biology, 15, 1-42.

GREGORIUS, H.-R. 1982b. The relationship between genic and genotypic fitnesses in diploid Protectedness of a biallelic polymorphism. Journal of Theoretical Biology, in print.

GREGORIUS, H.-R. 1982b. The relationship between genic and genotypic fitnesses in diploid populations. 96, 689-705 evolutionary theory, in press.

GREGORIUS, H.-R., AND ROSS, M. D. 1981. Selection in plant populations of effectively infinite size: I. Realized genotypic fitnesses. Mathematical Biosciences, 54, 291-307.

HAYMAN, B. I. 1953. Mixed selfing and random mating when homozygotes are at a disadvantage. Heredity, 7, 185-192. 
HUEHN, M. 1980. Equilibria of populations with selection and mixed selfing and random mating - with some implications for plant breeding. Ann. Amélior. Plantes, 30, 45-51. JAIN, S. K., AND WORKMAN, P. L. 1967. Generalized F-statistics and the theory of inbreeding and selection. Nature, 214, 674-678.

KIMURA, M., AND OHTA, T. 1971. Theoretical aspects of population genetics. Princeton University Press, Princeton, N.J.

LEWONTIN, R. C. 1958. A general method for investigating the equilibrium of gene frequency in a population. Genetics, 43, 421-433.

PROUT, T. 1968. Sufficient conditions for multiple niche polymorphisms. American Naturalist, $102,493-496$.

PURSER, A. F. 1966. Increase in heterozygote frequency with differential fertility. Heredity, $21,322-347$.

WALLACE, B. 1958. The comparison of observed and calculated zygotic distributions. Evolution, 12, 113-115.

WEIR, B. S. 1970. Equilibria under inbreeding and selection. Genetics, 65, 371-378.

WOEHRMANN, K., AND LANGE, P. 1970. Untersuchungen zur Wechselwirkung von Selektion und Selbstrungsrate auf das genetische Gleichgewicht unter besonderer Berücksichtigung tetraploider Populationen. II. Modell für diploide Populationen. Theoretical and Applied Genetics, 40, 289-295.

WORKMAN, P. L., AND JAIN, S. K. 1966. Zygotic selection under mixed random mating and self-fertilization: Theory and problems of estimation. Genetics, 54, 159-171.

zIEHE, M. 1981. Population trajectories for single locus additive fecundity selection and related selection models. Journal of Mathematical Biology, 11, 33-43.

ZIEHE, M. 1982. Genotypic frequencies of the offspring generation under selection on female or male gamete production in partially self-fertilizing plant populations. Göttingen Research Notes in Forest Genetics, 5, in press.

ZIEHE, M., AND GREGORIUS, H.-R. 1981. Deviations of genotypic structures from HardyWeinberg proportions under random mating and differential selection between the sexes. Genetics, 98, 215-230.

\section{APPENDIX 1}

\section{Convergence of genotypic structures}

(a) Selection on pollen production with $\mu_{11}=\mu_{22}<\mu_{12}$ and $\sigma \neq 1$.

Denoting $\mu_{11}, \mu_{22}$ by $\mu_{\mathrm{Hom}}$, the allelic frequency deviation of $A_{1}$ from 0.5 shows the following development over the generations:

$$
\left(p_{1}^{\prime}-0 \cdot 5\right)=\left[\frac{1+\sigma}{2}+\frac{(1-\sigma) \mu_{\text {Hom }}}{2 \bar{\mu}}\right]\left(p_{1}-0 \cdot 5\right) .
$$

$\mu_{\text {Hom }}=\bar{\mu}$, which is equivalent to $P_{12}=0$, is a possible constellation only for starting points. Thus except from fixation, we have $P_{12} \neq 0$ and $\mu_{\mathrm{Hom}}<\bar{\mu}$ after one generation at the latest. But this implies

$$
\frac{1+\sigma}{2}+\frac{1-\sigma}{2} \cdot \frac{\mu_{\text {Hom }}}{\bar{\mu}}<1
$$

so that $\left(p_{1}-0.5\right)$ is either a monotone decreasing (for $0.5<p_{1}$ ) or a monotone increasing (for $p_{1}<0.5$ ) or a constant (for $p_{1}=0.5$ ) sequence over the generations. Thus convergence of $p_{1}$ and $p_{2}$ to 0.5 follows and the limit set of genotypic trajectories except from fixation fulfils $\hat{p}_{1}=\hat{p}_{2}=$ $0 \cdot 5$. Considering those structures with $p_{1}=p_{2}=0 \cdot 5$, the heterozygote frequency changes in the following way:

$$
P_{12}^{\prime}=\frac{\sigma}{2} P_{12}+\frac{1-\sigma}{2},
$$

which leads to monotone convergence to an equilibrium in $(1-\sigma) /(2-\sigma)$. 
Therefore except from fixation all genotypic frequencies converge to $\hat{P}_{12}=$ $(2-\sigma) /(1-\sigma)$ with $\hat{p}_{1}=\hat{p}_{2}=0 \cdot 5$.

(b) Selection on ovule production with $\phi_{11}=\phi_{22}<\phi_{12}$ and $\sigma \neq 1$.

For this model

$$
\left(p_{1}-0 \cdot 5\right)=\left[\frac{1-\sigma}{2}+\frac{1+\sigma}{2} \frac{\phi_{\text {Hom }}}{\bar{\phi}}\right]\left(p_{1}-0 \cdot 5\right),
$$

where $\phi_{\mathrm{Hom}}=\phi_{11}=\phi_{22}$. As with (a) it is possible to show the convergence of allelic frequencies of both alleles to $0 \cdot 5$. However, for this model we have

$$
P_{12}^{\prime}=\frac{\sigma}{2} \frac{\phi_{12}}{\bar{\phi}} P_{12}+\frac{1-\sigma}{2} \text { for } \hat{p}_{1}=\hat{p}_{2}=0 \cdot 5 \text {. }
$$

There is exactly one equilibrium point with $\hat{p}_{1}=0.5$ and $P_{12}^{\prime}=P_{12}$. Defining $d=\left(\phi_{12}-\phi_{\mathrm{Hom}}\right) / \phi_{\mathrm{Hom}}$, this equilibrium reads

$$
\hat{P}_{12}=\left[(d+\sigma-2)+\sqrt{(d+\sigma-2)^{2}+8(1-\sigma) d}\right] / 4 d .
$$

It is easy to prove the monotone convergence of $P_{12}$ to $\hat{P}_{12}$ for $\hat{p}_{1}=0.5$.

(c) Differential viability and fertility selection symmetrical in the sexes, with $\left(s_{\mathrm{HOm}}=\right) \mathbf{s}_{11}=\mathbf{s}_{22}<\mathbf{s}_{12}$ and $\sigma \neq 1$.

Now $\left(p_{1}^{\prime}-0 \cdot 5\right)=\left(s_{\text {Hom }} / \bar{s}\right)\left(p_{1}-0 \cdot 5\right)$, and for $p_{1}=p_{2}=0.5$

$$
P_{12}^{\prime}=\frac{\sigma}{2} \frac{s_{12}}{\bar{s}} P_{12}+\frac{1-\sigma}{2} \text {. }
$$

The proof of convergence uses the same arguments as presented in (b). The equilibrium is the same as for (b) with $d=\left(s_{12}-s_{\mathrm{Hom}}\right) / s_{\mathrm{Hom}}$.

\section{APPENDIX 2}

\section{Protectedness of alleles}

The following definition of protectedness of an allele (compare Prout, 1968 ) as an instability property of its fixation state is directly taken from Gregorius (1982a):

An allele is called protected if it cannot be lost from any state of the population where all possible genotypes are present with positive frequencies; moreover it is required that the allele cannot stay for an indefinite number of generations at arbitrarily low frequencies. A diallelic polymorphism is called protected if both alleles are protected. This implies in particular the instability of both fixation points.

The analysis investigates the behaviour of the multiplication rate $p_{i}^{\prime} / p_{i}$ near the fixation state of $\boldsymbol{A}_{\boldsymbol{i}}$.

An application of the result of Gregorius (1982a) to a one-locus diallelic model with differential free pollen and ovule production but with equal self-fertilization rates for all genotypes leads to the following conditions for protectedness. $A_{1}$ is protected, if

$$
\frac{\sigma}{2} \frac{\phi_{12}}{\phi_{22}}\left(\frac{1-\sigma}{2} \frac{\mu_{11}}{\mu_{22}}+\frac{1+\sigma}{2} \frac{\phi_{11}}{\phi_{22}}-1\right)>\left(1-\sigma \frac{\phi_{11}}{\phi_{22}}\right)\left(1-\frac{1-\sigma}{2} \frac{\mu_{12}}{\mu_{22}}-\frac{1+\sigma}{2} \frac{\phi_{12}}{\phi_{22}}\right)
$$


or if

$$
\sigma \frac{\phi_{11}}{\phi_{22}}+\frac{1-\sigma}{2} \frac{\mu_{12}}{\mu_{22}}+\frac{1}{2} \frac{\phi_{12}}{\phi_{22}}>2 .
$$

For the protectedness of $A_{2}$, interchange the indices 1 and 2.

The special case $\sigma=1$ of an obligatory self-fertilizing organism leads to a protected polymorphism if $\phi_{11}, \phi_{22}<\frac{1}{2} \phi_{12}$. 